\title{
Patient selection and targeted treatment in the management of platinum-resistant ovarian cancer
}

This article was published in the following Dove Press journal:

Pharmacogenomics and Personalized Medicine

24 September 2013

Number of times this article has been viewed

\author{
Christopher P Leamon' \\ Chandra D Lovejoy ${ }^{2}$ \\ Binh Nguyen ${ }^{3}$ \\ 'Research and Development, \\ ${ }^{2}$ Regulatory Affairs, ${ }^{3}$ Clinical Affairs, \\ Endocyte, Inc., West Lafayette, IN, \\ USA
}

Correspondence: Christopher P Leamon Endocyte, Inc., 3000 Kent Avenue, West Lafayette, IN 47906, USA

$\mathrm{Tel}+\mathrm{I} 7654637175$

Fax + I 765463927 I

Email chrisleamon@endocyte.com

\begin{abstract}
Ovarian cancer (OC) has the highest mortality rate of any gynecologic cancer, and patients generally have a poor prognosis due to high chemotherapy resistance and late stage disease diagnosis. Platinum-resistant OC can be treated with cytotoxic chemotherapy such as paclitaxel, topotecan, pegylated liposomal doxorubicin, and gemcitabine, but many patients eventually relapse upon treatment. Fortunately, there are currently a number of targeted therapies in development for these patients who have shown promising results in recent clinical trials. These treatments often target the vascular endothelial growth factor pathway (eg, bevacizumab and aflibercept), DNA repair mechanisms (eg, iniparib and olaparib), or they are directed against folate related pathways (eg, pemetrexed, farletuzumab, and vintafolide). As many targeted therapies are only effective in a subset of patients, there is an increasing need for the identification of response predictive biomarkers. Selecting the right patients through biomarker screening will help tailor therapy to patients and decrease superfluous treatment to those who are biomarker negative; this approach should lead to improved clinical results and decreased toxicities. In this review the current targeted therapies used for treating platinum-resistant OC are discussed. Furthermore, use of prognostic and response predictive biomarkers to define OC patient populations that may benefit from specific targeted therapies is also highlighted.
\end{abstract}

Keywords: platinum-resistant ovarian cancer, targeted therapy, patient selection, folate receptor, VEGF, biomarkers

\section{Introduction}

Each year, more patients die of epithelial ovarian cancer (OC) than of any other gynecologic malignancy. Worldwide, $\mathrm{OC}$ is in the top ten of newly diagnosed cancer cases and in the top ten of mortality rates from cancer in women. ${ }^{1}$ In the US alone, OC was estimated to be newly diagnosed in 22,240 women in 2013 , leading to 14,030 deaths in that year. ${ }^{2}$

Most OC patients are only diagnosed at later stages, ie, when the disease is already well advanced, thereby complicating or limiting therapeutic options. The standard treatment for epithelial OC consists of aggressive debulking surgery followed by platinum-based chemotherapy. Although initial response to chemotherapy is approximately $70 \%$, the majority of these patients will unfortunately relapse. Therefore, OC has an overall poor prognosis and a low 5-year survival rate, especially in the case of platinum-resistant disease, due to its high treatment resistance (defined as disease recurrence within a treatment-free interval of less than 6 months). ${ }^{3}$ 


\section{Standard chemotherapies for treatment of platinum- resistant ovarian cancer}

There are currently four standard chemotherapy regimens used for patients who become resistant to platinum: paclitaxel, topotecan, pegylated liposomal doxorubicin (PLD), and gemcitabine.

\section{Paclitaxel}

Paclitaxel is a taxane that can stabilize microtubules to inhibit cell division. The drug was approved for treatment of recurrent OC when response rates (RR) of $25 \%$ to $37 \%$ were observed in multiple Phase II trials testing the 3-weekly schedule. ${ }^{4-6}$ In the study by Thigpen et al, a median progression-free survival (PFS) of 4.2 months and an overall survival (OS) of 16 months were observed. ${ }^{5}$ A Phase II trial showed that weekly dosing could lead to a $20.9 \%$ RR in platinum- and paclitaxel-resistant OC patients. ${ }^{7}$ This alternative weekly dosing schedule for paclitaxel was studied in many trials in refractory, persistent, or recurrent $\mathrm{OC}$ patients, as reviewed by Baird et al. ${ }^{8} \mathrm{~A}$ randomized Phase III study comparing weekly versus 3-weekly paclitaxel in recurrent OC patients (of whom half were platinum-resistant) showed no difference in RR, PFS, or OS. However, the weekly schedule had a better safety profile than the 3-weekly schedule, as considerably less neutropenia, neuropathy, and myalgia were observed. ${ }^{9}$ Of note, the occurrence of neutropenia was also reduced when paclitaxel was infused over 3 hours instead of 24 hours. $^{10}$ A recent randomized Phase II clinical trial (CARTAXHY) tested the efficacy of weekly paclitaxel as a single agent, or in combination with carboplatin, or weekly topotecan in patients with platinum-resistant OC. The results showed that the combination treatments increased hypersensitivity reactions, febrile neutropenia, and anemia, and did not improve RR or median PFS when compared to single agent weekly paclitaxel. ${ }^{11}$

\section{Topotecan}

Topotecan's mechanism of action is different from that of paclitaxel, as it does not directly block cell division, but instead induces irreversible DNA damage. Topotecan inhibits topoisomerase 1, leading to both single and double stranded DNA breaks that eventually promote apoptosis. Topotecan (administered once daily the first 5 days of 21-day cycles) was approved for treatment of OC after failure of initial or subsequent chemotherapy. This approval was based on a Phase III trial that showed it to be at least as effective as paclitaxel, with RR of $21 \%$ versus $13 \%$, and median PFS of 23 weeks versus 14 weeks, respectively. ${ }^{12}$
Unfortunately, topotecan treatment led to severe bone marrow suppression with $80 \%$ grade 4 neutropenia, $25 \%$ grade 4 thrombocytopenia, and $41 \%$ grade 3 or 4 anemia. ${ }^{12}$

As such toxicities are often dose limiting, multiple clinical trials have studied alternative dosing schedules to improve the tolerability of topotecan treatment. ${ }^{13-15}$ For example, one Phase II trial tested the effect of the "standard" dosing of topotecan $\left(1.5 \mathrm{mg} / \mathrm{m}^{2}\right.$, daily the first 5 days of 21-day cycles) compared with an alternative dosing regimen $\left(1.75 \mathrm{mg} / \mathrm{m}^{2}\right.$, once a week for 4 weeks, repeated every 6 weeks) in patients with recurrent $\mathrm{OC}$. The alternative dosing regimen led to a lower RR ( $9.6 \%$ compared with $22.6 \%$ in the standard dosing arm), but also decreased myelotoxicity (52\% of patients had grade 3 or 4 granulocytopenia in comparison with $94 \%$ in the standard dosing arm). ${ }^{14} \mathrm{~A}$ subsequent Phase II trial tested the effect of yet another dosing schedule $\left(1.5 \mathrm{mg} / \mathrm{m}^{2}\right.$, daily the first 3 days of 21 -day cycles). ${ }^{15}$ Compared to historical controls, this alternative dosing regimen seemed to decrease the toxicity of topotecan. In a meta-analysis of various clinical trials, it was concluded that modification of the topotecan dose, and potentially the dosing schedule, can indeed reduce hematologic toxicity without decreasing the efficacy of the drug. ${ }^{13}$

\section{PLD}

PLD is another standard chemotherapy regimen used for treating platinum-resistant OC. The active component of this drug (doxorubicin) is an anthracycline that intercalates DNA, leading to inhibition of replication and, subsequently, the inhibition of proper cell division. Efficacy of PLD in platinum-resistant OC has been confirmed in several Phase II trials. In detail, the trial by Muggia et al reported a $26 \%$ RR, median PFS of 5.7 months, and OS of 11 months. ${ }^{16}$ A subsequent trial showed a $17 \%$ RR and median PFS of 4.5 months. ${ }^{17}$ Of note, a Phase III trial testing topotecan treatment versus PLD treatment showed a trend toward a higher RR in the platinum-resistant OC patient subset treated with PLD, although there was no improvement of PFS or OS..$^{18,19}$

\section{Gemcitabine}

Gemcitabine could be considered an alternative to PLD for treatment of platinum-resistant OC. Gemcitabine is a nucleoside analog that can incorporate into the DNA, leading to a halt of DNA replication and induction of apoptosis. As the drug in combination with carboplatin had already been approved for treatment of recurrent platinum-sensitive OC, the efficacy of gemcitabine compared with PLD was tested 
in platinum-resistant OC patients as well..$^{3,20,21}$ The Phase III trial by Mutch et al, testing gemcitabine versus PLD in platinum-resistant OC, showed comparable median PFS (3.6 versus 3.1 months, respectively), median OS (12.7 versus 13.5 months), and overall RR (9.2\% versus $11.7 \%) .{ }^{20}$ Another Phase III trial testing the same drugs in OC patients who experienced treatment failure or disease recurrence/ progression within 12 months of the primary treatment confirmed that the efficacy of gemcitabine therapy was comparable to PLD. ${ }^{21}$

\section{Additional chemotherapies tested for treatment of platinum-resistant ovarian cancer}

The glutathione analog prodrug canfosfamide can be activated by glutathione S-transferase P1-1, leading to the induction of apoptosis. ${ }^{22,23}$ As this enzyme is overexpressed in many types of cancers, including OC, it has been studied for treatment of platinum-resistant OC. ${ }^{24-26} \mathrm{~A}$ Phase III trial in patients with platinum-refractory or -resistant OC showed that canfosfamide treatment was well tolerated, but it led to a lower median PFS than PLD or topotecan. ${ }^{25}$ These results led to the early discontinuation of another Phase III trial testing PLD with or without canfosfamide in platinumresistant OC patients. ${ }^{26}$ Interestingly, the interim analysis of this trial showed a trend toward improved median PFS in the combination arm, but further studies have not been conducted.

Finally, two other chemotherapies tested for the treatment of platinum-resistant OC are the microtubule stabilizing agents, patupilone and ixabepilone. ${ }^{27,28}$ The stabilization of the microtubules in the cell by these drugs interferes with (tumor) cell division, therefore counteracting tumor growth. A Phase II trial testing ixabepilone in patients with recurrent or persistent platinum- and taxane-resistant primary OC (or peritoneal carcinoma) showed a RR of $14.3 \%$, median PFS of 4.4 months, and median OS of 14.8 months. ${ }^{27}$ Patupilone was compared with PLD in a Phase III trial in platinum-refractory or -resistant epithelial ovarian, primary fallopian tube, or primary peritoneal cancer patients. ${ }^{28}$ That trial showed comparable median OS of 13.2 months and 12.7 months, respectively, and median PFS of 3.7 months in both groups. However, patupilone treatment did lead to a higher RR than PLD treatment (15.5\% versus $7.9 \%){ }^{28}$

Use of the aforementioned therapeutics has changed the way this disease is managed. But the fact remains that there is limited utility of these agents for platinum-resistant OC, as many patients do not respond to these treatments or they quickly relapse following an initial response. Therefore, treatment of platinum-resistant $\mathrm{OC}$ patients is often considered to be palliative instead of potentially curative. ${ }^{3}$

\section{Targeted therapies for treatment of platinum-resistant ovarian cancer}

As with many other types of cancer, targeted therapies may represent the future of platinum-resistant OC treatment. As seen with standard chemotherapies, drug resistance will remain an issue that must be addressed to advance OC treatment. However, genetic profiling of tumors before and after a treatment escape will provide insight into the drug resistance mechanisms. Based on the outcome of these studies it may be possible to predict which combinations of targeted therapies (or chemotherapies) can prevent treatment escape.

Current approaches to targeted therapy for OC can be classified into different groups, with each therapy having its own mechanism of action as well as specific advantages (Figure 1). For example, some of these therapies target the vascular endothelial growth factor (VEGF) pathway (bevacizumab and aflibercept), whereas others are directed against DNA repair mechanisms (iniparib and olaparib), or the folate pathway (pemetrexed, farletuzumab, and vintafolide). An overview of the most important clinical trials testing these drugs for treatment of platinum-resistant OC (often in combination with chemotherapy) can be found in Table $1.20,21,29-37$

\section{Bevacizumab}

Bevacizumab is a humanized monoclonal antibody (mAb) directed against VEGF. This mAb binds to VEGF, which is present in the tumor microenvironment, and prevents this cytokine from binding to the VEGF receptor, where it can initiate the formation of new blood vessels. With tumor angiogenesis hampered, tumor burden and the production of malignant ascites are reduced. ${ }^{38}$

The potential of this biologic molecule for OC treatment was first demonstrated in a Phase II trial testing against persistent or recurrent epithelial OC or primary peritoneal cancer. This trial showed a $21 \%$ RR, PFS of 4.7 months, and OS of 16.9 months. ${ }^{29}$ Based on these intriguing results, a subsequent Phase III trial (AURELIA trial) was conducted where platinum-resistant OC patients received chemotherapy (weekly paclitaxel, topotecan, or PLD) with (arm 1) or without (arm 2) bevacizumab until disease progression or unacceptable toxicity. The RR for the two respective treatment arms was $30.9 \%$ versus $12.6 \%$, and 


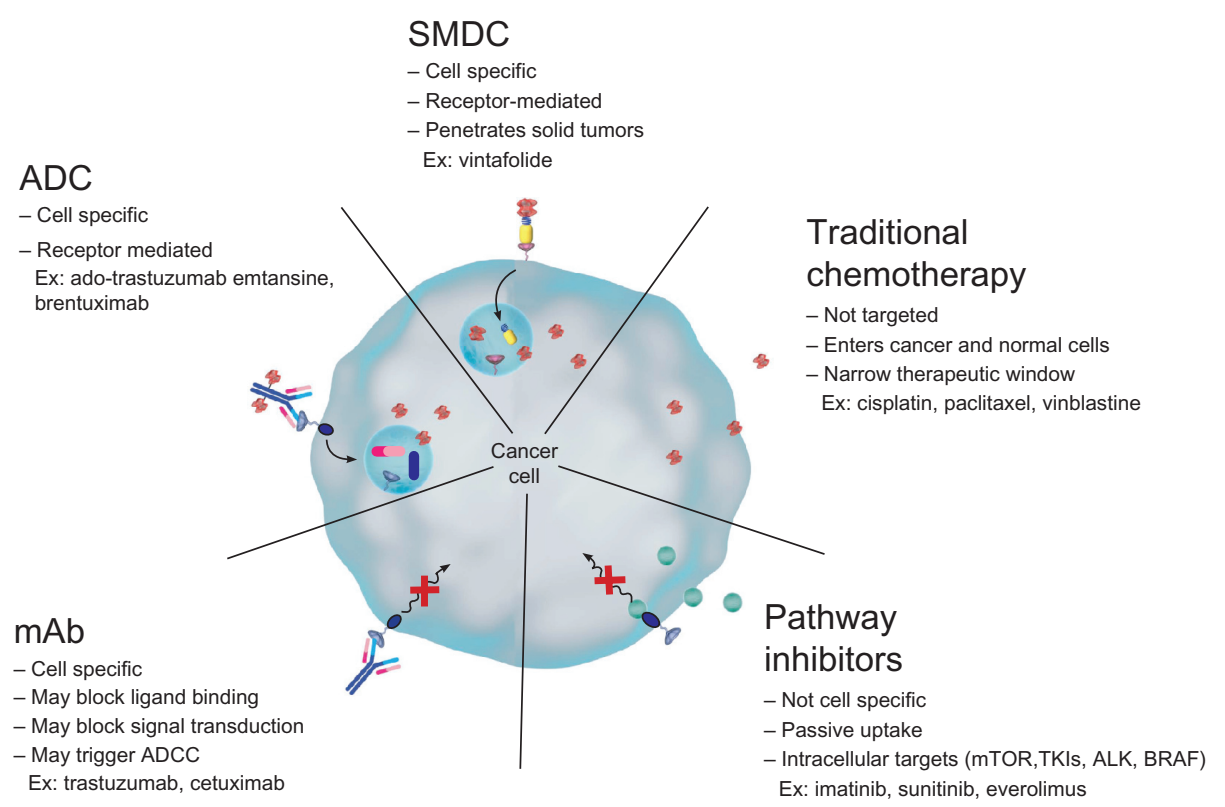

Figure I Overview of the different classes of ovarian cancer treatment.

Abbreviations: ADC, antibody-drug conjugate; ADCC, antibody dependent cellular toxicity; ALK, anaplastic lymphoma kinase; BRAF, v-Raf murine sarcoma viral oncogene homolog BI; Ex, example; mAb, monoclonal antibody; mTOR, mammalian target of rapamycin; SMDC, small molecule drug conjugate; TKI, tyrosine kinase inhibitor.

the median PFS was 6.7 versus 3.4 months (OS has not yet been reported). ${ }^{30}$ The adverse event (AE) profile of this drug can be concerning, though. Bevacizumab often leads to hypertension, arterial thromboembolic events, and minor bleedings. Furthermore, the drug has been reported to cause gastrointestinal complications such as perforations, which can be life threatening. ${ }^{39}$ Based on results from Phase III clinical trials (Gynecologic Oncology Group [GOG-128] and International Collaborative Ovarian Neoplasm Trials [ICON7]), bevacizumab in combination with standard

Table I Overview of selected therapies in development for treatment of ovarian cancer

\begin{tabular}{|c|c|c|c|c|c|c|c|}
\hline Study drug & Drug type & $\begin{array}{l}\text { Trial } \\
\text { phase }\end{array}$ & Patient group & $\mathbf{R R}, \%$ & $\begin{array}{l}\text { PFS (median), } \\
\text { months }\end{array}$ & $\begin{array}{l}\text { OS, } \\
\text { months }\end{array}$ & Reference \\
\hline Gemcitabine & Nucleoside analog & Phase III & Platinum-resistant $O C$ & 9.2 & 3.6 & 12.7 & Mutch et $\mathrm{al}^{20}$ \\
\hline Gemcitabine & Nucleoside analog & Phase III & $\begin{array}{l}\text { Platinum-resistant } O C \\
\text { (within } 12 \text { months) }\end{array}$ & 29 & 4.6 & 11.8 & Ferrandina et $\mathrm{al}^{2}$ \\
\hline Bevacizumab & VEGF antibody & Phase II & $\begin{array}{l}\text { Persistent or recurrent } \mathrm{OC} \text { or } \\
\text { primary peritoneal cancer }\end{array}$ & 21 & 4.7 & 16.9 & Burger et $\mathrm{al}^{29}$ \\
\hline $\begin{array}{l}\text { Bevacizumab } \\
\text { (plus standard CT) }\end{array}$ & VEGF antibody & Phase III & Platinum-resistant $O C$ & 30.9 & 6.7 & na & $\begin{array}{l}\text { Pujade- } \\
\text { Lauraine et al }{ }^{30}\end{array}$ \\
\hline $\begin{array}{l}\text { Aflibercept } \\
\text { (plus docetaxel) }\end{array}$ & VEGF decoy receptor & Phase II & $\begin{array}{l}\text { Recurrent OC, primary } \\
\text { peritoneal cancer, fallopian } \\
\text { tube cancer }\end{array}$ & 54 & 6.2 & 24.3 & Coleman et $\mathrm{al}^{31}$ \\
\hline Olaparib & PARP inhibitor & Phase II & $\begin{array}{l}B R C A \text { mutated platinum- } \\
\text { resistant or refractory } O C\end{array}$ & 40 & na & na & Fong et $\mathrm{al}^{32}$ \\
\hline Olaparib (high dose) & PARP inhibitor & Phase II & $B R C A$ mutated recurrent $O C$ & 31 & 8.8 & na & Kaye et $\mathrm{a}^{33}$ \\
\hline $\begin{array}{l}\text { Iniparib (plus } \\
\text { carboplatin, } \\
\text { gemcitabine) }\end{array}$ & PARP inhibitor & Phase II & $\begin{array}{l}\text { Platinum-resistant epithelial } \\
\text { OC, primary peritoneal } \\
\text { cancer, fallopian tube cancer }\end{array}$ & 31.6 & 5.9 & na & Birrer et $\mathrm{al}^{34}$ \\
\hline Pemetrexed & $\begin{array}{l}\text { Antifolate } \\
\text { antineoplastic agent }\end{array}$ & Phase II & $\begin{array}{l}\text { Platinum-resistant } \mathrm{OC} \text {, } \\
\text { primary peritoneal cancer }\end{array}$ & 21 & 2.9 & 11.4 & Miller et $\mathrm{a}^{35}$ \\
\hline Pemetrexed & $\begin{array}{l}\text { Antifolate } \\
\text { antineoplastic agent }\end{array}$ & Phase II & $\begin{array}{l}\text { Platinum-resistant } \mathrm{OC} \text {, } \\
\text { primary peritoneal cancer }\end{array}$ & 9.3 & 2.8 & 11.9 & Vergote et $\mathrm{al}^{36}$ \\
\hline Vintafolide & $\begin{array}{l}\text { SMDC of folate and } \\
\text { DAVLBH }\end{array}$ & Phase II & Platinum-resistant $O C$ & na & 5 & na & Naumann $2013^{37}$ \\
\hline
\end{tabular}

Abbreviations: CT, chemotherapy; DAVLBH, desacetylvinblastine hydrazide; na, not available; OC, ovarian cancer; OS, overall survival; PARP, poly (ADP-ribose) polymerase; PFS, progression free survival; RR, response rate; SMDC, small molecule drug conjugate; VEGF, vascular endothelial growth factor. 
chemotherapy was approved for treatment of platinumsensitive and first line OC, but not platinum-resistant $\mathrm{OC}$, in Europe. ${ }^{40,41}$ To date, bevacizumab has not been approved in the US.

\section{Aflibercept}

Another drug targeting the VEGF pathway is aflibercept, which is a recombinant decoy receptor fusion protein designed to sequester soluble VEGF-A, VEGF-B, and placental growth factor to prevent receptor docking and growth promoting signaling. In addition to regression of tumor associated vasculature, aflibercept treatment also inhibits new tumor vessel growth and remodeling/normalization of surviving tumor vasculature. Therefore, tumor burden and ascites formation are reduced upon treatment. ${ }^{42}$

Clinical efficacy of this drug was demonstrated in a Phase II trial testing the combination of docetaxel and aflibercept in patients with recurrent $\mathrm{OC}$, primary peritoneal cancer, or fallopian tube cancer. This trial reported a $54 \% \mathrm{RR}$, median PFS of 6.2 months, and OS of 24.3 months. ${ }^{31,43}$ Since hypertension, proteinuria, and bleeding are often associated with aflibercept treatment (ie, similar to bevacizumab), the safety profile of aflibercept is consistent with the class of agents targeting VEGF and the VEGF receptor, and remains a concern. ${ }^{44}$

\section{Iniparib}

The poly (ADP-ribose) polymerase (PARP) inhibitors, such as iniparib, represent another type of targeted therapy, specifically for the treatment of patients with $B R C A$ mutated OC. These drugs can block the PARP complex, leading to a disturbance of single stranded DNA repair. ${ }^{45}$ Subsequently, the cells are more prone to develop double strand injuries that require homologous recombination to be repaired. As patients with a $B R C A$ mutation have impaired homologous recombination activity, tumor cells from these patients are not able to repair the DNA damage and, consequently, undergo apoptosis.

The PARP inhibitor iniparib has been tested in clinical trials as monotherapy or in combination with chemotherapy. ${ }^{46}$ Results from the Phase II trial (NCT00677079) in which iniparib was tested as a monotherapy in $B R C A$ mutation positive relapsed or refractory OC or primary peritoneal cancer patients have not yet been reported. However, a Phase II trial testing the efficacy of iniparib combined with carboplatin and gemcitabine in platinum-resistant epithelial $\mathrm{OC}$, fallopian tube cancer, or primary peritoneal carcinoma patients did yield promising results, showing a RR of $31.6 \%$ and median PFS of 5.9 months. ${ }^{34}$ In this trial, patients were not selected for the $B R C A$ mutation. However, $B R C A$ selection may not be required, as it was shown in recent studies that iniparib interacts only nonspecifically with the PARP proteins and, therefore, may have a different mechanism of action than previously thought. ${ }^{47}$

\section{Olaparib}

Olaparib seems to be the most clinically advanced PARP inhibitor for treatment of OC. The potential of olaparib has been tested in multiple clinical trials in both platinumsensitive and -resistant $\mathrm{OC}$ patients with or without $B R C A$ mutations. ${ }^{46}$ A Phase II trial in which patients with $B R C A$ mutated platinum-resistant or platinum-sensitive $\mathrm{OC}$ were treated with the PARP inhibitor olaparib showed a 40\% RR. ${ }^{32}$ Another Phase II trial in recurrent OC patients with $B R C A$ mutations showed, respectively, 33\% RR and 13\% RR, when patients received olaparib at $400 \mathrm{mg}$ twice daily, or $100 \mathrm{mg}$ twice daily, suggesting that higher dosing of olaparib may be more effective. ${ }^{48}$ The results from a Phase II trial in which recurrent $\mathrm{OC}$ patients with a $B R C A$ mutation were treated with low dose olaparib (200 mg, twice daily), high dose olaparib (400 mg, twice daily), or PLD were in line with this finding. ${ }^{33}$ This trial reported, for the three respective treatment arms, RRs of $25 \%, 31 \%$, and $18 \%$ and median PFS of 6.5 months, 8.8 months, and 7.1 months. ${ }^{33}$ Based on the promising results in Phase II trials, a Phase III trial testing olaparib as a first line treatment will be performed in BRCA-mutated OC patients (NCT01844986).

Although PARP inhibitor treatments can be effective against platinum-resistant $\mathrm{OC}$, they appear to produce higher RRs in platinum-sensitive OC patients. ${ }^{3}$ Importantly, the toxicity profile of the PARP inhibitors in patients with a $B R C A$ mutation is favorable and primarily consists of low grade gastrointestinal disorders and fatigue. ${ }^{32}$ However, when combined with chemotherapeutic agents, PARP inhibitors like olaparib seem to increase bone marrow toxicity (thrombocytopenia, anemia, neutropenia) to levels greater than that observed with the drugs alone. ${ }^{49}$ Given the promising clinical activity of PARP inhibitors, this class of drugs will be further developed for the treatment of both platinum-sensitive and -resistant OC.

\section{Pemetrexed}

Folate is necessary to synthesize, repair, and methylate DNA, as it is involved in the synthesis of purines and pyrimidines. As most tumors have an excessive growth rate, cancer cells generally have a concomitant need for folate. 
The most common way by which folate is transported into a nonmalignant cell is through the reduced folate carrier (RFC). ${ }^{50}$ However, some cells express a distinct proton-coupled folate transporter (PCFT) which can also transport folates. Pemetrexed is an antifolate antineoplastic agent that can enter cells via these transporters. ${ }^{51}$ Upon entry to the cytoplasm, the drug is converted to a polyglutamated form, which has an increased retention half-life within malignant cells. While in the cytosol, pemetrexed can inhibit a variety of folate dependent enzymes to interrupt DNA replication and other folate dependent reactions. ${ }^{52,53}$

At the early stages of its development, pemetrexed treatment was shown to cause occasional serious myelosuppression (in some cases, leading to death) and gastrointestinal disorders. ${ }^{54}$ However, supplemental use of daily oral folic acid was later found to reduce or prevent some of these serious AEs to enable continued clinical development. ${ }^{56,55}$ Generally, the safety profile of pemetrexed consists of both hematologic (anemia, leukopenia, neutropenia) and nonhematologic AEs (fatigue, vomiting, and ascites formation). ${ }^{35,36}$

Two Phase II trials have evaluated the efficacy of pemetrexed treatment in platinum-resistant OC patients. ${ }^{35,36}$ One of these trials tested pemetrexed as second line cytotoxic treatment of persistent or recurrent platinum-resistant $\mathrm{OC}$ or primary peritoneal cancer, and the results showed a $21 \% \mathrm{RR}$, PFS of 2.9 months, and OS of 11.4 months. ${ }^{35}$ The other trial compared standard versus high dose pemetrexed in platinumresistant OC and primary peritoneal cancer. ${ }^{36}$ The RR in that study was $9.3 \%$ for the standard dose treatment arm and $10.4 \%$ for the high dose treatment arm. The median PFS was 2.8 months in both arms and OS was 11.9 and 10.3 months for the standard and high dose arm, respectively. ${ }^{36}$ Although these results were encouraging, pemetrexed is not widely used with platinum-resistant $\mathrm{OC}$ patients.

\section{Farletuzumab}

Folate can also enter a cell via endocytosis mediated by a high affinity folate receptor (FR).$^{50}$ This process is often used by OC cells, as they (and many other types of tumor cells) frequently overexpress FR-alpha $(\mathrm{FR} \alpha) .{ }^{55} \mathrm{FR}$ expression is much less frequent in nonmalignant cells, which rely mostly on the RFC and PCFT instead of the FR to capture folates. ${ }^{58}$ The FR forms an ideal target for many targeted therapies because overexpression is quite specific for tumor cells.

Farletuzumab is a humanized mAb targeting $\mathrm{FR} \alpha$. When the antibody is present on the cell surface, the cell is recognized by antibody dependent cell mediated cytotoxicity and complement dependent cytotoxicity immune mechanisms. These processes result in death of those cells expressing the FR. ${ }^{59,60}$

To date, farletuzumab has mainly been tested in platinum-sensitive OC patients. Nevertheless, one Phase I trial enrolling platinum-resistant OC patients showed that the drug was generally safe, well tolerated, and led to stable disease in $36 \%$ of patients, but farletuzumab did not lead to objective responses. ${ }^{61}$ Unfortunately, a Phase III trial testing farletuzumab treatment for platinum-resistant OC was recently terminated because it did not meet prespecified criteria for continuation of that trial (NCT00738699).

\section{Vintafolide}

Vintafolide (EC145; Endocyte, Inc., West Lafayette, IN, USA) represents a distinct form of targeted therapy, one that is directed against the FR but also employs a different mechanism of action than either pemetrexed or farletuzumab. ${ }^{62}$ Vintafolide is a small molecule drug conjugate (SMDC) consisting of folic acid linked to the vinca alkaloid desacetylvinblastine hydrazide (DAVLBH), which is a potent microtubule destabilizing agent (Figure 2) ${ }^{63}$ An advantage of this drug is that the linker mechanism of the SMDC allows for drug stability in the blood. Furthermore, vintafolide is a unique type of targeted therapy, as the active DAVLBH "warhead" is released upon endocytosis. ${ }^{64}$ Since most nonmalignant cells do not express high levels of the FRs, this drug has a favorable safety profile. Vintafolide specifically targets cells that overexpress the FR, such as OC cells, as this receptor has high affinity for the folic acid component in vintafolide. Thus, vintafolide "targets" the DAVLBH warhead specifically to FR-expressing cells (Figure 3).

A recent Phase II trial (PRECEDENT) in platinumresistant OC patients compared PLD (arm 1) versus vintafolide combined with PLD (arm 2). ${ }^{27}$ The median PFS was 11.7 versus 21.7 weeks for the two respective treatment arms (hazard ratio [HR] 0.626, $P=0.031)$. Drug related serious adverse events and the number of patients discontinuing treatment because of at least one drug related serious adverse event was not

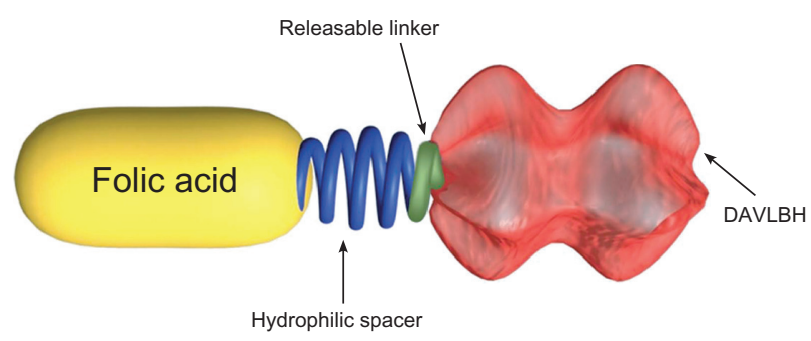

Figure 2 Chemical construction of vintafolide as a small molecule drug conjugate. Abbreviation: DAVLBH, desacetylvinblastine hydrazide. 


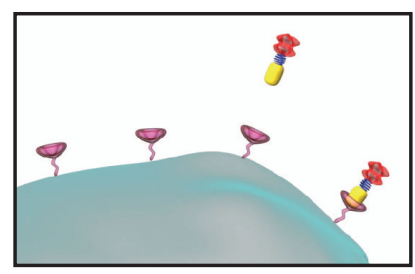

Vintafolide binds the folate receptor

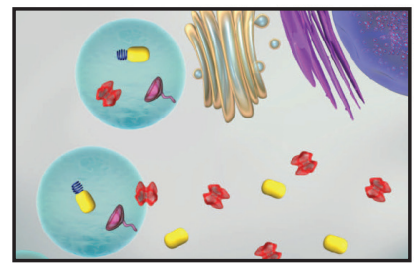

DAVLBH escapes the endosome and exerts activity on cell

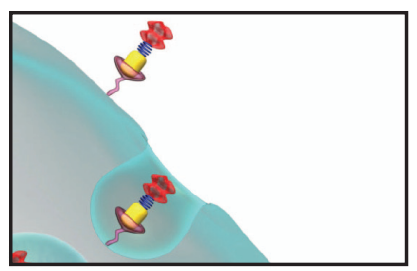

Vintafolide is internalized via endocytosis

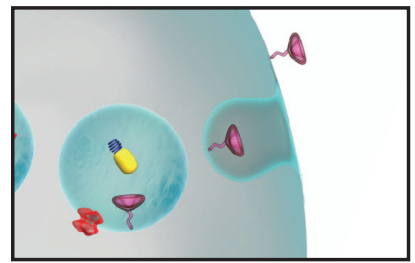

$\mathrm{FR}$ recycles back to the cell surface

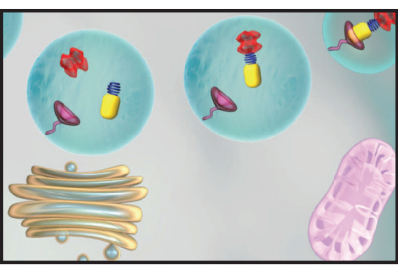

Vintafolide's linker is cleaved in the endosome to release DAVLBH

Figure 3 Overview of mechanism of action of vintafolide.

Abbreviations: DAVLBH, desacetylvinblastine hydrazide; FR, folate receptor.

statistically different between treatment arms; this result was expected since vintafolide is a tissue targeted agent. For patients who received vintafolide combined with PLD, some AEs (leukopenia, neutropenia, peripheral sensory neuropathy, and abdominal pain) were reported at a higher incidence than for patients who received PLD only. ${ }^{37}$ However, vintafolide treatment was well tolerated overall. The PRECEDENT trial included an optional scan using etarfolatide (EC20; Endocyte, Inc., West Lafayette, IN, USA), a technetium-labeled, folatetargeted companion imaging agent that can anatomically identify FR positive tumors (Figure 4). ${ }^{65-67}$ Results showed that in $40 \%$ of the screened platinum-resistant OC patients, all target tumor lesions were found to be etarfolatide (ie, FR) positive, and the subanalysis of this patient population showed that vintafolide treatment improved PFS even further, from 6.6 to 24 weeks (HR 0.381, $P=0.013$ ) for arms 1 and 2 , respectively. ${ }^{37}$ Importantly, PFS for arm 1 was much lower in this subset of patients when compared to the intent to treat (ITT) population because FR expression in OC is a negative prognostic indicator. ${ }^{68}$

\section{Patient selection - the age of predictive medicine Use of response predictive biomarkers in cancer treatment}

The goal in cancer therapy is to treat only those patients who may actually benefit from a specific treatment. Patient selection using response predictive biomarkers is already applied in management of certain tumor types, such as breast cancer (trastuzumab treatment) and melanoma (vemurafenib treatment).

For example, as human epidermal growth factor receptor 2 (Her2) overexpression is a predictor of treatment effect, trastuzumab is indicated as a single agent or combined with paclitaxel, only for metastatic breast cancer patients who overexpress the Her2 protein. ${ }^{69}$ Her2 overexpression must therefore be confirmed by an approved test. Such tests either use fluorescence in situ hybridization to detect amplification of the Her2 gene, or immunohistochemistry to visualize increased expression of Her2 protein. In another example, the $\mathrm{BRAF}^{\mathrm{V} 600 \mathrm{E}}$ inhibitor vemurafenib was shown to yield high response rates in melanomas that had been shown to express $B R A F^{V 600 E}$ (a mutated signaling protein that supports tumor cell proliferation and survival), but not in melanomas that express wildtype $B R A F{ }^{71}$ Therefore, the drug was approved only for metastatic melanoma patients who are positive for the $B R A F^{V 600 E}$ mutation. ${ }^{70}$ The approved test for this drug $\left(\operatorname{cobas}^{\circledR} 4800\right.$ BRAF V600 Mutation Test, Roche Molecular Systems Inc.) uses polymerase chain reaction to detect $B R A F^{V 600 E}$ mutations in DNA extracted from formalin fixed paraffin embedded tissues.

Such response predictive biomarkers are often used as prognostic biomarkers. In many cases the biomarker has a negative prognostic value which, consequently, renders it as an attractive target for therapy. Returning to the aforementioned examples, Her2 was determined to be both a prognostic and response predictive biomarker for breast 
Step 1: Etarfolatide whole body scan
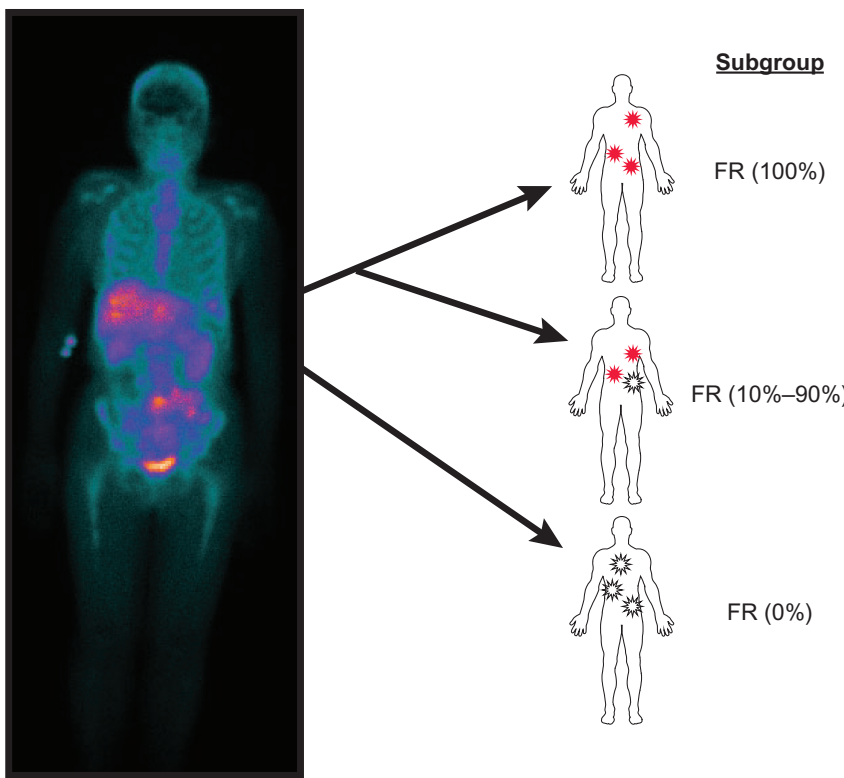

Step 2: Define threshold

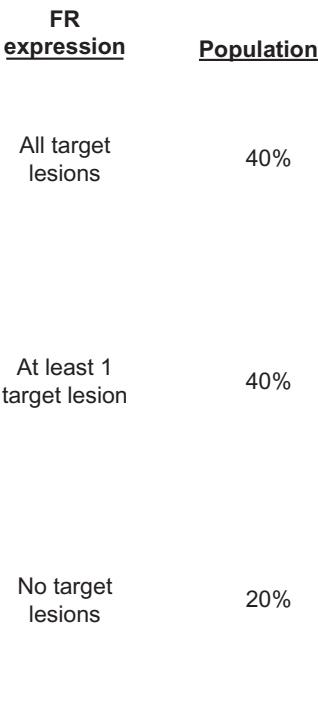

Figure 4 Overview of how etarfolatide can be used to subcategorize patients. Abbreviation: FR, folate receptor.

cancer patients; thus, Her2 positive patients have a shorter time to disease relapse and a lower OS. ${ }^{72}$ Likewise, BRAF ${ }^{\mathrm{V} 600 \mathrm{E}}$ is also both a prognostic and response predictive biomarker; this particular $B R A F$ mutation increases the risk of mortality by 1.7 fold in melanoma patients ( $95 \%$ confidence interval $1.39-2.12){ }^{73}$

Many of the targeted therapies for the treatment of platinum-resistant OC lead to an objective response in only a subset of patients. The identification of response predictive biomarkers for these OC targeted therapies is required to (1) avoid superfluous treatment since they will help select patients who could benefit from the targeted therapy, and (2) prevent biomarker negative patients from exposure to intensive and sometimes toxic treatments. Both these advantages benefit the patient, and the costs to the health care system could be reduced.

\section{Cancer antigen I25 (CA-I25) as biomarker for ovarian cancer}

To optimize OC treatment, biomarkers with very direct links to the targeted therapies are currently being studied. ${ }^{74} \mathrm{CA}-125$ is one such biomarker. CA-125 is a membrane glycoprotein commonly found on cells in the female reproductive tract, and its expression level seems to be linked to cellular growth. Serum levels of this protein are elevated in more than $80 \%$ of OC patients $;^{74}$ hence, serum CA-125 concentration has become a well known indicator for measuring OC disease progression. Unfortunately, the response predictive and prognostic value of this marker remains unclear. Many studies have shown that rising serum CA-125 levels anticipate disease relapse by about 3 months; therefore, the level of this protein is regularly measured in OC patients while in remission. ${ }^{75}$ Furthermore, the serum levels of CA-125 are often used as a surrogate endpoint for disease progression in OC clinical trials. Although the added value of CA-125 monitoring is currently under debate, the need for early detection of disease recurrence remains crucial.

There are a number of conflicting reports on the prognostic value of elevated CA-125 levels at baseline, but preoperative expression of CA-125 appears to be a negative prognostic factor for survival. ${ }^{74}$ Furthermore, CA-125 serum level is potentially an independent prognostic factor for achieving a pathologic complete response upon chemotherapy, with patients with lower serum levels experiencing longer PFS. ${ }^{74}$ Although CA-125 serum levels are strongly correlated to disease progression and, to a lesser extent, prognosis, this protein is not targeted by any type of approved therapy, as it is not believed to have a direct role in the etiology of OC. ${ }^{74}$

\section{VEGF as biomarker for ovarian cancer}

VEGF has been detected both in surgical samples and in sera of OC patients, and high preoperative serum VEGF was shown to be an independent negative prognostic factor in patients with all stages of OC. ${ }^{74,76}$ As mentioned above, the VEGF targeted drugs, bevacizumab and aflibercept, are promising targeted therapies for the treatment of platinum-resistant OC. 
Thus, one would expect that VEGF is a response predictive biomarker for these treatments. If so, increased clinical responses to these drugs would be expected in patients who have high VEGF serum levels. However, a recent study found that baseline VEGF serum levels do not seem to be predictive of a response to bevacizumab treatment. ${ }^{77}$ Overall, VEGF does not appear to be a relevant biologic indicator for OC, particularly in terms of patient selection or predictive medicine, but rather a target for therapy that is more indicative of the pathobiology of OC.

\section{Biomarkers for PARP inhibitor treatment}

Considering the mechanism of action of PARP inhibitors, patients carrying the $B R C A$ mutation might be expected to have a better response to treatment. Indeed, a Phase II trial testing olaparib in patients with $\mathrm{OC}$ or triple negative breast cancer showed a $41 \% \mathrm{RR}$ in those patients with the $B R C A$ mutation, and a RR of only $24 \%$ in patients without the $B R C A$ mutation. ${ }^{78}$ This finding implies that the $B R C A$ mutation may indeed be used as a response predictive biomarker for PARP inhibitor treatment. As PARP inhibitors undergo further clinical development for treatment of $\mathrm{OC}$, this correlation will merit further investigation.

\section{Folate receptor expression as a biomarker for ovarian cancer}

Although CA-125 and VEGF expression have prognostic value for the survival of OC patients, to date their value as response predictive biomarkers is rather limited. The lack of response predictive biomarkers makes it challenging to select patients who may benefit the most from targeted therapies. In contrast, tumor FR expression appears to be both a prognostic biomarker for OC patients as well as a response predictive biomarker for targeted therapies directed against the FR.

As discussed previously, folate can enter cells by either the RFC, PCFT, or by FR mediated endocytosis. ${ }^{50,51}$ The RFC is expressed on most cells but has only a low affinity for folate. The PCFT has more limited normal tissue expression, but it too has low affinity for folates under physiologic $\mathrm{pH} .{ }^{58}$ In contrast, FRs have a high affinity for folate (especially folic acid), and they are expressed on many types of epithelial cancer cells while usually being absent on nonmalignant cells. The reason for FR upregulation in malignancies is poorly understood, but it is speculated to be related to the tumor's increased need for folate due to higher metabolic activity and increased rate of nucleotide biosynthesis. The FR does not appear to drive development of OC, but FR overexpression is associated with tumor progression. ${ }^{55}$
Furthermore, its expression has a negative impact on OS of serous OC patients. ${ }^{68}$

Considering the potentially diverse mechanisms of action of FR targeted agents, increased clinical responses in patients who express the FR on their tumor cells may be expected. Thus, having the ability to screen and select only those patients who have FR positive tumors would be highly valuable, especially in the context of real time anatomic screening using a noninvasive approach. Etarfolatide represents the most advanced screening method of this type. ${ }^{65-67,79}$ Etarfolatide is a peptidic analog of the high affinity folic acid molecule that efficiently chelates technetium-99m $\left({ }^{99 m} \mathrm{Tc}\right)$. Upon intravenous infusion, the small ${ }^{99 \mathrm{~m}}$ Tc-etarfolatide molecule rapidly distributes within all tissues of the body and specifically binds to FR positive tissues. Based on its low molecular weight and high water solubility characteristics, unbound ${ }^{99 \mathrm{~m} T c-e t a r f o l a t i d e ~ i s ~}$ rapidly cleared from the body (principally through the kidneys) to allow for single photon emission computed tomography based detection of FR positive tumors in as little as an hour (Figure 4).

${ }^{99 \mathrm{~m}}$ Tc-etarfolatide was used in a single arm Phase II trial in which patients with recurrent or persistent epithelial ovarian, fallopian tube, or primary peritoneal carcinoma were first imaged to establish their "FR status," followed by treatment with the folate targeted therapeutic vintafolide. ${ }^{80}$ Patients were assigned to one of three categories: FR (100\%) (all target lesions FR positive), FR (10\%-90\%) (at least one, but not all target lesions FR positive), or FR ( $0 \%$ ) (no FR positive target lesions). The results showed that vintafolide had the greatest efficacy when $100 \%$ of a patient's target lesions were FR positive, as the PFS for FR (100\%) patients was longer for FR (10\%-90\%) patients. The disease control rate and OS were also higher in the FR $(100 \%)$ patients when compared with FR (10\%-90\%) patients. ${ }^{76,80}$

Based on these initial results, a randomized Phase II trial (PRECEDENT) was conducted, and the results supported what was observed in the single agent Phase II study in that the positive effect of vintafolide on clinical endpoints was more pronounced in patients with FR positive lesions. ${ }^{37}$ Thus, in all patients, regardless of FR status of the target lesions (ie, the ITT population), vintafolide treatment combined with PLD treatment improved median PFS when compared with PLD treatment alone. However, when specifically analyzing FR (100\%) patients, the median PFS improved even more. Notably, the lower median PFS after PLD treatment of FR (100\%) patients compared to the ITT population is most likely related to the fact that the 
degree of FR expression is associated with signs of biologic aggressiveness of OC. ${ }^{81}$

Both Phase II trials have shown that using response predictive biomarkers (eg, FR expression) may help to select subsets of patients who will benefit the most from a given treatment. As discussed previously, patient selection can improve the outcome of a clinical trial, and it helps to prevent superfluous treatment of patients. Unfortunately, for platinum-resistant OC there are currently (apart from FR expression) not many response predictive biomarkers available for targeted therapies.

\section{The implications of predictive medicine to the drug approval process}

The many benefits obtained from utilizing a diagnostic agent for patient selection are readily apparent. Regulators, pharmaceutical companies, payers, and patients have all proselytized the importance and value of targeted drug development. Obvious benefits include the potential ability to ensure a greater likelihood that patients benefit from the treatments administered, reduction of clinical trial size and duration, and increase in overall observed efficacy, thus maximizing efficiency of drug development and improving treatment options for patients. The merit of this development path achieves particular importance in situations where the drug/diagnostic target is associated with a poorer patient prognosis (as is the case with Her2 for breast cancer, and now for FR in OC). In these situations where the targeted patient subset has no means for selection or treatment, as well as a worse overall outcome on standard therapies, the targeted development path becomes imperative for cancer patient care.

Although the medical need and benefit to patients are clear, targeted drug development also presents its regulatory challenges. For example, the current thinking on development paths and viable registrational trial designs are in a state of flux and evolution as regulations, guidance, and policy attempt to keep pace with rapid scientific developments. Of note, in just the last year, new Food and Drug Administration (FDA) guidance was issued and the FDA has recently presented the new regulatory option of "Breakthrough Therapy Designation" as a means for providing more flexibility and openness to the novel paradigm presented by targeted drug development. ${ }^{82}$ However, the path to approval is always filled with risk, and doing so in an evolving regulatory landscape while seeking approval of two new agents (eg, one therapeutic and one diagnostic) that must be coordinated between different FDA divisions in the US, as well as multiple global agencies, presents a development path that is largely uncharted. The goals and merits of this new direction are easily recognized, but the lack of regulatory clarity provides sufficient challenges to merit careful consideration for those seeking to utilize the targeted therapy approach. Recent approvals of crizotinib and vemurafenib demonstrate the potential for success and provide a sense of optimism for the possibility of additional targeted therapies, such as vintafolide and etarfolatide, to provide benefit to cancer patients with high unmet need.

\section{Future outlook}

For an increasing number of tumors, biomarker screening is used to select patients who can benefit from a targeted therapy. As discussed, Her2 expression is a prerequisite for trastuzumab treatment of breast cancer patients; likewise, $B R A F^{V 600 E}$ expression is a requirement for vemurafenib treatment of melanoma patients. For OC, two Phase II trials using vintafolide and its companion diagnostic agent, ${ }^{99 \mathrm{~m}} \mathrm{Tc}-$ etarfolatide, have confirmed the added value of combining biomarker screening with a tumor targeted therapy.

In the future, prescreening of a patient's FR status using etarfolatide may also be a companion diagnostic tool for other FR targeted agents. One such agent that is currently in preclinical development is EC1456, which is an SMDC of folic acid linked to the highly potent antimitotic agent tubulysin B. ${ }^{83}$ This SMDC has been shown in vitro to specifically inhibit the growth of FR positive cells, and its potency and specificity were confirmed in multiple human xenograft tumor models, including OC (Endocyte, Inc., unpublished data 2012). Such targeted therapies can provide substantial therapeutic benefit using dosing regimens that are well tolerated due to the tissue specificity of the drug. Another FR targeted agent that might be usefully combined with etarfolatide screening as a diagnostic tool is farletuzumab. However, this drug has shown little meaningful clinical activity. The mechanism of action of the two FR targeted agents (targeted delivery of chemotherapy versus immune response induction) may contribute to the difference in clinical efficacy, as the FR does seem to be a relevant clinical target in OC.

Biomarker screening, combined with a targeted therapy, is also being developed for other malignancies. For example, in prostate cancer the ${ }^{9 \mathrm{~mm}} \mathrm{Tc}$-labeled imaging agent EC0652 is currently being used to visualize prostate-specific membrane antigen (PSMA) positive tumors (Endocyte, Inc., unpublished data 2013). It is also anticipated that PSMA positive patients may then move to therapy using an agent 
like EC1169, a PSMA targeted SMDC constructed with the tubulysin warhead. ${ }^{84}$

Currently, there are no other regimens under development for OC using a biomarker screening test for application of a companion targeted therapy. But identification of other pharmaceutical "pairs" will become increasingly important for further advancement of personalized therapies for platinum-resistant OC treatment.

\section{Acknowledgments}

All authors are employees of Endocyte, Inc., West Lafayette, IN, USA. Editorial and medical writing assistance was provided by Anna Hooijkaas, TRM Oncology, The Hague, the Netherlands, the work was partially funded by Endocyte, Inc., West Lafayette, IN, USA.

\section{Disclosure}

All authors report no conflict of interest in this work.

\section{References}

1. Ferlay J, Shin HR, Bray F, Forman D, Mathers C, Parkin DM. GLOBOCAN 2008 v2.0, Cancer Incidence and Mortality Worldwide: IARC CancerBase No 10 [homepage on the Internet]. Lyon, France: International Agency for Research on Cancer; 2010. Available from: http:// globocan.iarc.fr. Accessed April 22, 2013.

2. Siegel R, Naishadham D, Jemal A. Cancer statistics, 2013. CA Cancer J Clin. 2013;63(1):11-30.

3. Naumann RW, Coleman RL. Management strategies for recurrent platinum-resistant ovarian cancer. Drugs. 2011;71(11):1397-1412.

4. McGuire WP, Rowinsky EK, Rosenshein NB, et al. Taxol: a unique antineoplastic agent with significant activity in advanced ovarian epithelial neoplasms. Ann Int Med. 1989;111(4):273-279.

5. Thigpen JT, Blessing JA, Ball H, Hummel SJ, Barrett RJ. Phase II trial of paclitaxel in patients with progressive ovarian carcinoma after platinum-based chemotherapy: a Gynecologic Oncology Group study. J Clin Oncol. 1994;12(9):1748-1753.

6. Rowinsky EK, Donehower RC. Paclitaxel (taxol). $N$ Engl J Med. 1995;332(15):1004-1014.

7. Gynecologic Oncology Group, Markman M, Blessing J, et al. Phase II trial of weekly paclitaxel $\left(80 \mathrm{mg} / \mathrm{m}^{2}\right)$ in platinum and paclitaxel-resistant ovarian and primary peritoneal cancers: a Gynecologic Oncology Group study. Gynecol Oncol. 2006;101(3):436-440.

8. Baird RD, Tan DS, Kaye SB. Weekly paclitaxel in the treatment of recurrent ovarian cancer. Nat Rev Clin Oncol. 2010;7(10):575-582.

9. Rosenberg P, Andersson H, Boman K, et al. Randomized trial of single-agent paclitaxel given weekly versus every three weeks and with peroral versus intravenous steroid premedication to patients with ovarian cancer previously treated with platinum. Acta Oncol. 2002;41(5): $418-424$.

10. Eisenhauer EA, ten Bokkel Huinink WW, et al. European-Canadian randomized trial of paclitaxel in relapsed ovarian cancer: highdose versus low-dose and long versus short infusion. J Clin Oncol. 1994;12(12):2654-2666.

11. Lortholary A, Largillier R, Weber B, et al; GINECO group France. Weekly paclitaxel as a single agent or in combination with carboplatin or weekly topotecan in patients with resistant ovarian cancer: the CARTAXHY randomized phase II trial from Groupe d'Investigateurs Nationaux pour l'Etude des Cancers Ovariens (GINECO). Ann Oncol. 2012;23(2):346-352.
12. ten Bokkel Huinink W, Gore M, Carmichael J, et al. Topotecan versus paclitaxel for the treatment of recurrent epithelial ovarian cancer. J Clin Oncol. 1997;15(6):2183-2193.

13. Armstrong DK. Topotecan dosing guidelines in ovarian cancer: reduction and management of hematologic toxicity. Oncologist. 2004;9(1):33-42.

14. Hoskins P, Eisenhauer E, Beare S, et al. Randomized phase 11 study of two schedules of topotecan in previously treated patients with ovarian cancer: a National Cancer Institute of Canada Clinical Trials Group study. J Clin Oncol. 1998;16(6):2233-2237.

15. Markman M, Kennedy A, Webster K, Kulp B, Peterson G, Belinson J. Phase 2 evaluation of topotecan administered on a 3-day schedule in the treatment of platinum- and paclitaxel-refractory ovarian cancer. Gynecol Oncol. 2000;79(1):116-119.

16. Muggia FM, Hainsworth JD, Jeffers S, et al. Phase II study of liposomal doxorubicin in refractory ovarian cancer: antitumor activity and toxicity modification by liposomal encapsulation. J Clin Oncol. 1997;15(3): 987-993.

17. Gordon AN, Granai CO, Rose PG, et al. Phase II study of liposomal doxorubicin in platinum- and paclitaxel-refractory epithelial ovarian cancer. J Clin Oncol. 2000;18(17):3093-3100.

18. Gordon AN, Tonda M, Sun S, Rackoff W; Doxil Study 30-49 Investigators. Long-term survival advantage for woman treated with pegylated liposomal doxorubicin compared with topotecan in a phase 3 randomized study of recurrent and refractory epithelial ovarian cancer. Gynecol Oncol. 2004;95(1):1-8.

19. Gordon AN, Fleagle JT, Guthrie D, Parkin DE, Gore ME, Lacave AJ. Recurrent epithelial ovarian carcinoma: a randomized phase III study of pegylated liposomal doxorubicin versus topotecan. J Clin Oncol. 2001;19(14):3312-3322.

20. Mutch DG, Orlando M, Goss T, et al. Randomized phase III trial of gemcitabine compared with pegylated liposomal doxorubicin in patients with platinum-resistant ovarian cancer. J Clin Oncol. 2007;25(19): 2811-2818.

21. Ferrandina G, Ludovisi M, Lorusso D, et al. Phase III trial of gemcitabine compared with pegylated liposomal doxorubicin in progressive or recurrent ovarian cancer. J Clin Oncol. 2008;26(6):890-896.

22. Satyam A, Hocker MD, Kane-Maguire KA, Morgan AS, Villar HO, Lyttle MH. Design, synthesis, and evaluation of latent alkylating agents activated by glutathione S-transferase. J Med Chem. 1996;39(8): 1736-1747.

23. Lyttle MH, Satyam A, Hocker MD, et al. Glutathione-S-transferase activates novel alkylating agents. J Med Chem. 1994;37(10):1501-1507.

24. Laborde E. Glutathione transferases as mediators of signaling pathways involved in cell proliferation and cell death. Cell Death Differ. 2010;17(9):1373-1380.

25. Vergote I, Finkler N, del Campo J, et al; ASSIST-1 Study Group. Phase 3 randomised study of canfosfamide (Telcyta ${ }^{\circledR}$, TLK286) versus pegylated liposomal doxorubicin or topotecan as third-line therapy in patients with platinum-refractory or -resistant ovarian cancer. Eur J Cancer. 2009;45:2324-2332.

26. Vergote I, Finkler NJ, Hall JB, et al. Randomized phase III study of canfosfamide in combination with pegylated liposomal doxorubicin compared with pegylated liposomal doxorubicin alone in platinumresistant ovarian cancer. Int J Gynecol Cancer. 2010;20(5): 772-780.

27. De Geest K, Blessing JA, Morris RT, et al. Phase II clinical trial of ixabepilone in patients with recurrent or persistent platinum- and taxane-resistant ovarian or primary peritoneal cancer: a gynecologic oncology group study. J Clin Oncol. 2010;28(1):149-153.

28. Colombo N, Kutarska E, Dimopoulos M, et al. Randomized, openlabel, phase III study comparing patupilone (EPO906) with pegylated liposomal doxorubicin in platinum-refractory or -resistant patients with recurrent epithelial ovarian, primary fallopian tube, or primary peritoneal cancer. J Clin Oncol. 2012;30(31):3841-3847.

29. Burger RA, Sill MW, Monk BJ, Greer BE, Sorosky JI. Phase II trial of bevacizumab in persistent or recurrent epithelial ovarian cancer or primary peritoneal cancer: a Gynecologic Oncology Group Study. J Clin Oncol. 2007;25(33):5165-5171. 
30. Pujade-Lauraine E, Hilpert F, Weber B, et al. AURELIA: A randomized phase III trial evaluating bevacizumab (BEV) plus chemotherapy (CT) for platinum (PT)-resistant recurrent ovarian cancer (OC). J Clin Oncol. 2012;30(Suppl 15):abstract LBA5002.

31. Coleman RL, Duska LR, Ramirez PT, et al. Phase II multi-institutional study of docetaxel plus aflibercept (AVE0005, NSC\# 724770) in patients with recurrent ovarian, primary peritoneal, and fallopian tube cancer. $J$ Clin Oncol. 2011;29(Suppl 15): Abstract 5017.

32. Fong PC, Yap TA, Boss DS, et al. Poly(ADP)-ribose polymerase inhibition: frequent durable responses in BRCA carrier ovarian cancer correlating with platinum-free interval. J Clin Oncol. 2010;28(15): 2512-2519.

33. Kaye SB, Lubinski J, Matulonis U, et al. Phase II, open-label, randomized, multicenter study comparing the efficacy and safety of olaparib, a poly (ADP-ribose) polymerase inhibitor, and pegylated liposomal doxorubicin in patients with BRCA1 or BRCA2 mutations and recurrent ovarian cancer. J Clin Oncol. 2012;30(4):372-379.

34. Birrer MJ, Konstantinopoulos P, Penson RT, et al. A phase II trial of iniparib (BSI-201) in combination with gemcitabine/carboplatin (GC) in patients with platinum-resistant recurrent ovarian cancer. J Clin Oncol. 2011;29(Suppl): Abstract 5005.

35. Miller DS, Blessing JA, Krasner CN, et al. Phase II evaluation of pemetrexed in the treatment of recurrent or persistent platinum-resistant ovarian or primary peritoneal carcinoma: a study of the Gynecologic Oncology Group. J Clin Oncol. 2009;27(16):2686-2691.

36. Vergote I, Calvert H, Kania M, Kaiser C, Zimmermann AH, Sehouli J. A randomised, double-blind, phase II study of two doses of pemetrexed in the treatment of platinum-resistant, epithelial ovarian or primary peritoneal cancer. Eur J Cancer. 2009;45:1415-1423.

37. Naumann RW, Coleman RL, Burger RA, et al. PRECEDENT: a randomized phase II trial comparing EC145 and pegylated liposomal doxorubicin (PLD) in combination, versus PLD alone, in subjects with platinum-resistant ovarian cancer. $J$ Clin Oncol. 2013. In press.

38. Mabuchi S, Terai Y, Morishige K, et al. Maintenance treatment with bevacizumab prolongs survival in an in vivo ovarian cancer model. Clin Cancer Res. 2008;14(23):7781-7789.

39. Heitz F, Harter P, Barinoff J, et al. Bevacizumab in the treatment of ovarian cancer. Adv Ther. 2012;29(9):723-735.

40. Burger RA, Brady MF, Bookman MA, et al; Gynecologic Oncology Group. Incorporation of bevacizumab in the primary treatment of ovarian cancer. $N$ Engl J Med. 2011;365(26):2473-2483.

41. Perren TJ, Swart AM, Pfisterer J, et al; ICON7 Investigators. A phase 3 trial of bevacizumab in ovarian cancer. $N$ Engl J Med. 2011;365(26): 2484-2496.

42. Sharma T, Dhingra R, Singh S, et al. Aflibercept: A novel VEGF targeted agent to explore the future perspectives of anti-angiogenic therapy for the treatment of multiple tumors. Mini Rev Med Chem. 2013;13(4):530-540.

43. Coleman RL, Duska LR, Ramirez PT, et al. Phase 1-2 study of docetaxel plus aflibercept in patients with recurrent ovarian, primary peritoneal, or fallopian tube cancer. Lancet Oncol. 2011;12(12):1109-1117.

44. Tang PA, Cohen SJ, Kollmannsberger C, et al. Phase II clinical and pharmacokinetic study of aflibercept in patients with previously treated metastatic colorectal cancer. Clin Cancer Res. 2012;18(21): 6023-6031.

45. Curtin NJ. PARP inhibitors for cancer therapy. Expert Rev Mol Med. 2005;7(4):1-20.

46. Ratner ES, Sartorelli AC, Lin ZP. Poly (ADP-ribose) polymerase inhibitors: on the horizon of tailored and personalized therapies for epithelial ovarian cancer. Curr Opin Oncol. 2012;24(5):564-571.

47. Liu X, Shi Y, Maag DX, et al. Iniparib nonselectively modifies cysteinecontaining proteins in tumor cells and is not a bona fide PARP inhibitor. Clin Cancer Res. 2012;18(2):510-523.

48. Audeh MW, Carmichael J, Penson RT, et al. Oral poly(ADP-ribose) polymerase inhibitor olaparib in patients with BRCA1 or BRCA2 mutations and recurrent ovarian cancer: a proof-of-concept trial. Lancet. 2010;376.(9737):245-251.
49. Chen Y, Zhang L, Hao Q. Olaparib: a promising PARP inhibitor in ovarian cancer therapy. Arch Gynecol Obstet. Epub April 26, 2013.

50. Antony AC. The biological chemistry of folate receptors. Blood. 1992;79(11):2807-2820.

51. Zhao R, Goldman ID. The molecular identity and characterization of a Proton-coupled Folate Transporter - PCFT; biological ramifications and impact on the activity of pemetrexed. Cancer Metastasis Rev. 2007;26(1):129-139.

52. Mendelsohn LG, Shih C, Chen VJ, Habeck LL, Gates SB, Shackelford KA. Enzyme inhibition, polyglutamation, and the effect of LY231514 (MTA) in purine biosynthesis. Semin Oncol. 1999;26(2 Suppl 6):42-47.

53. Miotti S, Bagnoli M, Ottone F, Tomassetti A, Colnaghi MI, Canevari S. Simultaneous activity of two different mechanisms of folate transport in ovarian carcinoma cell lines. J Cell Biochem. 1997;65(4): 479-491.

54. Tomao F, Panici PB, Frati L, Tomao S. Emerging role of pemetrexed in ovarian cancer. Expert Rev Anticancer Ther. 2009;9(12):1727-1735.

55. Siu MK, Kong DS, Chan HY, et al. Paradoxical impact of two folate receptors, FR $\alpha$ and RFC, in ovarian cancer: effect on cell proliferation, invasion and clinical outcome. PloS One. 2012;7(11):e47201.

56. Niyikiza C, Baker SD, Seitz DE, et al. Homocysteine and methylmalonic acid: markers to predict and avoid toxicity from pemetrexed therapy. Mol Cancer Ther. 2002;1(7):545-552.

57. Adjei AA. Pemetrexed (ALIMTA), a novel multitargeted neoplastic agent. Clin Cancer Res. 2004;10(12 Pt):4276s-4280s.

58. Zhao R, Diop-Bove N, Visentin M, Goldman ID. Mechanisms of membrane transport of folates into cells and across epithelia. Annu Rev Nutr. 2011;31:177-201.

59. Kamen BA, Smith AK. Farletuzumab, an anti-folate receptor $\alpha$ antibody, does not block binding of folate or anti-folates to receptor nor does it alter the potency of anti-folates in vitro. Cancer Chemother Pharmacol. 2012;70(1):113-120.

60. Ebel W, Routhier EL, Foley B, et al. Preclinical evaluation of MORAb-003, a humanized monoclonal antibody antagonizing folate receptor-alpha. Cancer Immun. 2007;7:6.

61. Konner JA, Bell-McGuinn KM, Sabbatini P, et al. Farletuzumab, a humanized monoclonal antibody against folate receptor alpha, in epithelial ovarian cancer: a phase I study. Clin Cancer Res. 2010;16(1):5288-5295.

62. Dosio F, Milla P, Cattel L. EC-145, a folate-targeted Vinca alkaloid conjugate for the potential treatment of folate receptor-expressing cancers. Curr Opin Investig Drugs. 2010;11(12):1424-1433.

63. Vlahov IR, Santhapuram HK, Kleindl PJ, Howard SJ, Stanford KM, Leamon CP. Design and regioselective synthesis of a new generation of targeted chemotherapeutics. Part 1: EC145, a folic acid conjugate of desacetylvinblastine monohydrazide. Bioorg Med Chem Lett. 2006;16(19):5093-5096.

64. Vlahov IR, Leamon CP. Engineering folate-drug conjugates to target cancer: from chemistry to clinic. Bioconjug Chem. 2012;23(7): $1357-1369$

65. Leamon CP, Parker MA, Vlahov IR, et al. Synthesis and biological evaluation of EC20: a new folate-derived, $(99 \mathrm{~m})$ Tc-based radiopharmaceutical. Bioconjug Chem. 2002;13(6):1200-1210.

66. Reddy JA, Xu LC, Parker N, Vetzel M, Leamon CP. Preclinical evaluation of $(99 \mathrm{~m}) \mathrm{Tc}-\mathrm{EC} 20$ for imaging folate receptor-positive tumors. J Nucl Med. 2004;45(5):857-866.

67. Fisher RE, Siegel BA, Edell SL, et al. Exploratory study of 99mTc-EC20 imaging for identifying patients with folate receptor-positive solid tumors. J Nucl Med. 2008;49(6):899-906.

68. Chen YL, Chang MC, Huang CY, et al. Serous ovarian carcinoma patients with high alpha-folate receptor had reducing survival and cytotoxic chemo-response. Mol Oncol. 2012;6(3):360-369.

69. Herceptin [prescribing information]. South San Francisco, CA: Genentech, Inc; 2010. Available from: http://www.accessdata.fda.gov/ drugsatfda_docs/label/2010/103792s5250lbl.pdf. Accessed April 22, 2013. 
70. Zelboraf [prescribing information]. South San Francisco, CA: Genentech, Inc; 2011. Available from: http://www.accessdata.fda.gov/drugsatfda_ docs/label/2011/202429s000lbl.pdf. Accessed April 22, 2013.

71. Chapman PB, Hauschild A, Robert C, et al. Improved survival with vemurafenib in melanoma with BRAF V600E mutation. $N$ Engl J Med. 2011;364(26):2507-2516.

72. Ross JS, Fletcher JA. The HER-2/neu oncogene in breast cancer: prognostic factor, predictive factor, and target for therapy. Oncologist. 1998;3(4):237-252.

73. Safaee Ardekani G, Jafarnejad SM, Tan L, Saeedi A, Li G. The prognostic value of BRAF mutation in colorectal cancer and melanoma: a systematic review and meta-analysis. PLoS One. 2012;7(10):e47054.

74. Gadducci A, Cosio S, Tana R, Genazanni AR. Serum and tissue biomarkers as predictive and prognostic variables in epithelial ovarian cancer. Crit Rev Oncol Hematol. 2009;69(1):12-27.

75. Verheijen RH, Cibula D, Zola P, et al; Council of the European Society of Gynaecologic Oncology. Cancer antigen 125: lost to follow-up? a European society of gynaecological oncology consensus statement Int J Gynecol Cancer. 2012;22(1):170-174.

76. Hefler LA, Zeillinger R, Grimm C, et al. Preoperative serum vascular endothelial growth factor as a prognostic parameter in ovarian cancer. Gynecol Oncol. 2006;103(2):512-517.

77. Madsen CV, Steffensen KD, Olsen DA, et al. Serial measurements of serum PDGF-AA, PDGF-BB, FGF2, and VEGF in multiresistant ovarian cancer patients treated with bevacizumab. J Ovarian Res. 2012;5(1):23.

78. Gelmon KA, Tischkowitz M, Mackay H, et al. Olaparib in patients with recurrent high-grade serous or poorly differentiated ovarian carcinoma or triple-negative breast cancer: a phase 2, multicenter, open-label, non-randomised study. Lancet Oncol. 2011;12(9):852-861.
79. Edelman MJ, Harb WA, Pal SE, et al. Multicenter trial of EC145 in advanced, folate-receptor positive adenocarcinoma of the lung. JThorac Oncol. 2012;7(10):1618-1621.

80. Kuo P, Naumann RW, Symanowski J, et al. 99 mTc-etarfolatide (EC20) SPECT imaging for the identification of ovarian and non-small cell lung cancer patients who are most likely to benefit from folate-receptortargeted agent vintafolide (EC145). AACR-SNMMI Joint Conference on State-of-the-Art Molecular Imaging in Cancer and Biology Therapy; February 27-March 2, 2013; San Diego, CA, USA. abstract 41.

81. Toffoli G, Cernigoi C, Russo A, Gallo A, Bagnoli M, Boiocchi M. Overexpression of folate binding protein in ovarian cancers. Int $J$ Cancer. 1997;74(2):193-198.

82. Fox JL. FDA moves on breakthrough therapies. Nat Biotechnol. 2013;31(5):374.

83. Leamon CP, Reddy JA, Wang Y, et al. EC0531: A potent folate-targeted tubulysin conjugate. Proceedings of the 101st Annual Meeting of the American Association for Cancer Research; April 17-21, 2010; Washington, DC. Philadelphia (PA): AACR; Cancer Res 2010; 70(Suppl 8):abstract nr 4414.

84. Reddy JA, Bloomfield A, Dorton R, et al. PSMA-specific anti-tumor activity of the targeted-tubulysin conjugate, EC1169. Proceedings of the 104th Annual Meeting of the American Association for Cancer Research; April 6-10, 2013; Washington, DC, USA. Philadelphia (PA): AACR; 2013.
Pharmacogenomics and Personalized Medicine

\section{Publish your work in this journal}

Pharmacogenomics and Personalized Medicine is an international, peerreviewed, open access journal characterizing the influence of genotype on pharmacology leading to the development of personalized treatment programs and individualized drug selection for improved safety, efficacy and sustainability. This journal is indexed on the American Chemical

\section{Dovepress}

Society's Chemical Abstracts Service (CAS). The manuscript management system is completely online and includes a very quick and fair peer-review system, which is all easy to use. Visit http://www.dovepress. $\mathrm{com} /$ testimonials.php to read real quotes from published authors. 